\title{
ENFOQUE SISTÉMICO APLICADO AL MANEJO DE PAROUES METROPOLITANOS, UNA POSICIÓN DESDE BOGOTÁ D.C. - COLOMBIA
}

\section{SYSTEMIC APPROACH APPLIED TO THE MANAGEMENT OF PARKS METROPOLITAN, A POSITION FROM BOGOTA, COLOMBIA}

\begin{abstract}
Adriana Posada Arrubla ${ }^{1}$, Ángela Daniela Paredes Buitrago ${ }^{2}$, Geovanny Esteban Ortiz Romero ${ }^{2}$
${ }^{1}$ Economista Agrícola, Esp. Gestión Social y Ambiental, M.Sc. Planeación Urbana y Regional, Docente-Investigadora, Facultad Ingenierías. Universidad de Ciencias Aplicadas y Ambientales U.D.C.A, calle 222 No. 55-37, Bogotá D.C., e-mail: aposada@udca.edu.co; ${ }^{2}$ Auxiliares de investigación U.D.C.A, Ingenieros Geógrafos y Ambientales. Universidad de Ciencias Aplicadas y Ambientales U.D.C.A, calle 222 No. 55-37, Bogotá D.C., e-mail: andapabu14@ hotmail.com; geor0306@hotmail.com
\end{abstract}

Rev. U.D.C.A Act. \& Div. Cient. 19(1): 207-217, Enero-Junio, 2016

\section{RESUMEN}

Los grandes parques urbanos son un distintivo fundamental del espacio público natural y construido de una ciudad; además, hacen parte de la estructura ecológica principal, que define un ambiente urbano. Aun así, su manejo ambiental no es integrado es reduccionista y enfocado al mantenimiento de las áreas recreacionales, aislándolo de otras dinámicas ambientales, que permiten su funcionamiento. Se presenta aquí, una alternativa de análisis, con base en el enfoque sistémico, que permite el planteamiento de un modelo de manejo ambiental, en este caso, para el parque metropolitano Simón Bolívar, de la capital colombiana, propuesta que implica la asociación de variables funcionales, con el fin de optimizar y de potenciar su buen uso y aprovechamiento, al considerarlo como unidad interrelacionada con sistemas de mayor jerarquía y complejidad y, a la vez, su funcionamiento depende de subsistemas, que condicionan su comportamiento interno.

Palabras clave: Parque Metropolitano Simón Bolívar, Teoría de Sistemas, Manejo Ambiental, Ambiente Urbano.

\section{SUMMARY}

Large urban parks are a banner of the built and natural public space in a city, moreover, they are part of the main ecological structure that defines an urban environment. Even so, their environmental management is not integrated, it is reductionist and focused on the maintenance of the recreational areas, isolating it from other socio-environmental dynamics that allow its operation. Presented here, is an alternative of analysis based on the systemic approach, which allows the approach of a model of environmental management, in this case, for the Metropolitan Park Simón Bolívar in the Colombian capital. Proposal involving the association of socio-environmental variables in order to optimize and enhance their good use and exploitation, to consider it as a unit connected with higher hierarchy and complexity, systems and at the same time, its performance depends on subsystems affecting their internal behavior.

Key words: Simon Bolivar Metropolitan Park, Systems Theory, Environmental Management, Urban Environment.

\section{INTRODUCCIÓN}

La expansión de las ciudades, por el incontrolable crecimiento poblacional, trae consigo el crecimiento sectorizado de la economía, avances de infraestructura y, al mismo tiempo, una paulatina reducción de las áreas naturales, lo cual, pone en juego su sostenibilidad ambiental. Para Fernández (2002), una ciudad es un ambiente urbano, porque es la relación sociedad/naturaleza, luego de la transformación de espacios naturales, que genera un problema ambiental, por exceso de carga de demandas de consumo social, sobre el soporte territorial. Es el caso de Bogotá, donde se requiere la aplicación de medidas que equilibren cargas, que permitan la conectividad de los cuerpos naturales y, de esta manera, aporten en el mejoramiento de la calidad ambiental de la capital colombiana. Según Gómez (2005), la calidad ambiental incide en la calidad de vida urbana que, de acuerdo con Maldonado (1999), son condiciones óptimas que conjugan y determinan sensaciones de confort en lo biológico y psicosocial, vinculadas a un determinado grado de satisfacción de servicios y de percepción del espacio habitable, como sano, 
seguro y grato; sin embargo, en Colombia, la política de gestión ambiental urbana (MAVDT, 2008) destaca las dificultades respecto a la calidad del hábitat de las áreas urbanas en el país, derivado de la reducción, invasión, deterioro o pérdida de los componentes del espacio público. A pesar de ello, el espacio público es estructurante de la ciudad y, al mismo tiempo, regulador de sus condiciones ambientales (AMB, 1998), puesto que, al ser un bien colectivo, en él confluyen la mayoría de las dinámicas sociales desarrolladas por la ciudadanía, por lo cual, es pertinente destacar la utilidad de las áreas que optimizan el hábitat urbano. Es el caso de los parques urbanos que, debido a su multifuncionalidad, de conservación natural, recreativa, cultural, educativa, esparcimiento y paisajística (García, 1989), se convierten en escenarios fundamentales para los ciudadanos y, en síntesis, son áreas públicas, que rompen el esquema urbanizador, cumpliendo un rol socio- ecológico (García \& Guerrero, 2006).

En Bogotá, los parques urbanos, de escala metropolitana y zonal, son administrados por el Instituto Distrital de Recreación y Deporte (en adelante, I.D.R.D.), que promueve su buen uso, orientado principalmente en la recreación, el aprovechamiento del tiempo libre y la actividad física; no obstante, la gestión articulada se queda corta, pues la integralidad de variables es mínima. Para enfatizar en esta dificultad, la unidad de estudio del presente trabajo es el "Parque Metro- politano Simón Bolívar" (en adelante, P.M.S.B), que además de ser considerado el pulmón en medio de la urbanización capitalina, es un espacio con oferta multifuncional para los habitantes de todas las localidades de la ciudad, debido a su importancia. Este parque urbano es un estandarte del espacio público y hace parte de la Estructura Ecológica Principal del Distrito, que definen el ambiente urbano (engranaje de factores sociales y entorno natural) de la capital colombiana. Aun así, este parque no cuenta con un manejo ambiental integrado, por lo que se opta por un manejo reduccionista y enfocado únicamente en el mantenimiento de las áreas naturales, aislándolo de las dinámicas sociales, que permiten el funcionamiento del parque.

Para Cardona (1996), el débil manejo de carácter ambiental impide la armonía en los elementos que componen el parque y la ejecución de medidas para satisfacer las funciones básicas. Atendiendo a esta situación, Ángel \& Velásquez (s.f.) reflexionan sobre el hecho urbano en su perspectiva ambiental, ya que conduce, necesariamente, a entender la dinámica relacional de un sistema complejo. Por ello, se debe entender el parque como unidad sistémica y sus interdependencias funcionales, de manera recíproca, con sistemas de mayor jerarquía y complejidad, como lo es la Estructura Ecológica Principal bogotana.

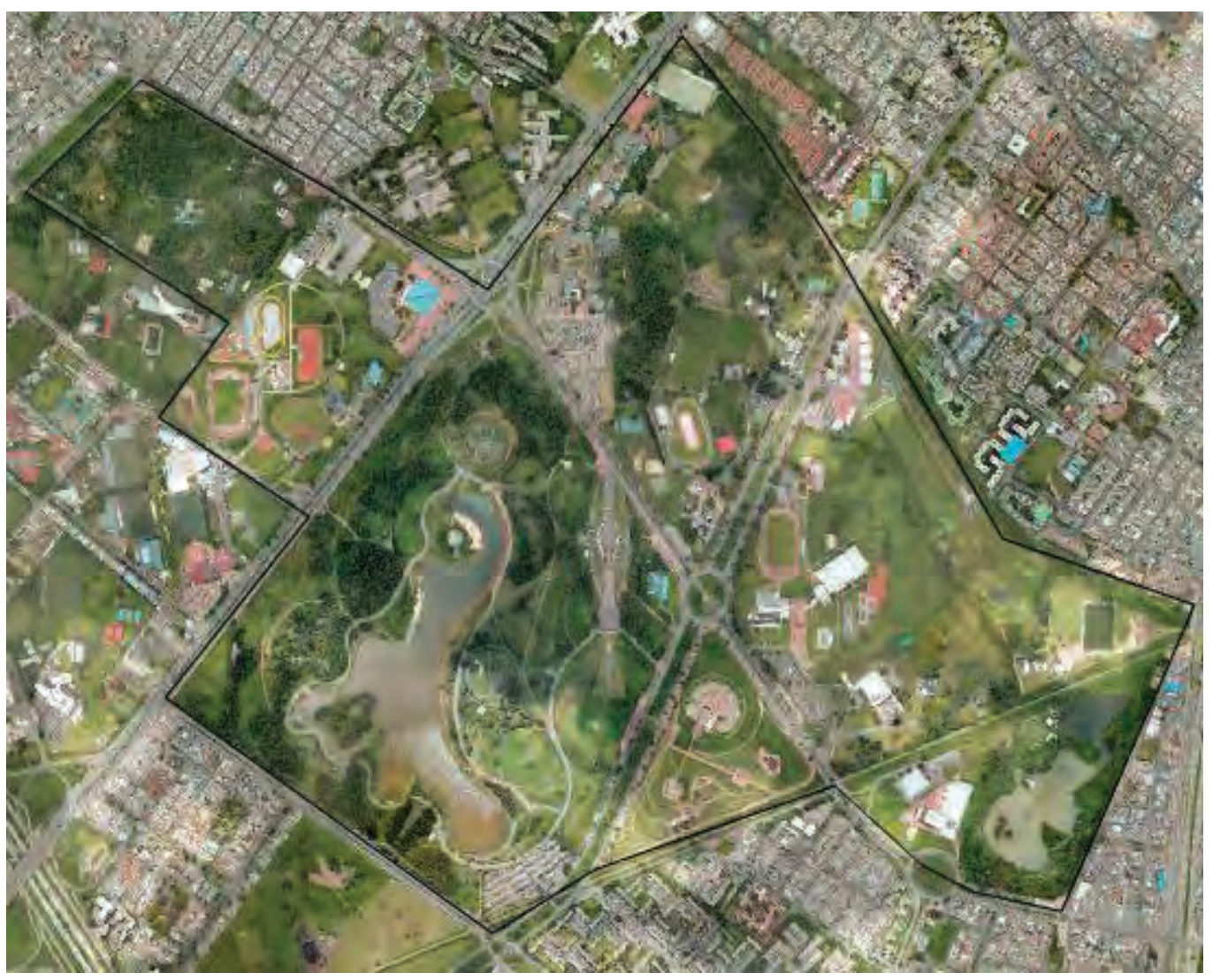

Figura 1. Parque Metropolitano Simón Bolívar, 2014. Fuente: Portal de Mapas de Bogotá IDECA. 
El objetivo de esta publicación consiste en mostrar cómo la unidad urbana "parque" se puede analizar bajo un enfoque sistémico, con la intención de aportar opciones para su manejo ambiental, teniendo en cuenta sus contribuciones funcionales al ambiente de la capital y de su entorno. Según la OMS (1965), los estudios basados en sistemas, ofrecen extraordinarias posibilidades para la organización urbana, ya que permiten asociar variables con alta complejidad. El Parque Metropolitano Simón Bolívar (Figura 1) hace parte de la red de parques administrados por el I.D.R.D., que cuenta con 370 hectáreas y está conformado por 14 sectores, cada uno de ellos con sus correspondientes escenarios (AMB, 2010). Existe, para el efecto, una motivación mundial, impulsada en Europa, para la gestión del territorio, desde las partes de la ciudad verde hacia un todo de bienestar común (CEA, 2014).

\section{MATERIALES Y MÉTODO}

Sobre el contexto de los parques, históricamente, se indagó sus administraciones, sus características generales y algunas con opción de ser especializadas, mediante el Software ArcGis, a partir de datos obtenidos de la IDECA (2015); la información secundaria, se obtuvo en libros y en revistas científicas, orientadas al componente geográfico. Para identificar aspectos puntuales, se revisaron trabajos y autores relacionados con los temas de manejo ambiental, parques urbanos, geografía sistémica y teoría general de sistemas, por medio de bases de datos, como Dialnet, Scielo y Ebsco. La recolección de información espacial, se logró en el Instituto Geográfico Agustín Codazzi y en imágenes satelitales de Google Earth, además de algunos planos proporcionados por el I.D.R.D.

Para la aplicación de la teoría general de sistemas, primero se estableció, como contexto general, el macrosistema del P.M.S.B., junto con sus componentes e interacciones; luego, se establecieron las bases para la elaboración del modelo, en donde se determinaron las propiedades y sus correspondientes conexiones e interacciones, aplicando la simbología (Odum, 1988).

\section{Desarrollo metodológico:}

Macrosistema: Para esta investigación, el sistema de mayor jerarquía es el ambiente urbano que, a su vez, está integrado por los sistemas naturales, antrópico y funcional, que son representados de la siguiente manera:

Sistema Natural: Representado en la estructura ecológica principal de Bogotá, compuesta por el sistema distrital de áreas protegidas, las áreas de especial importancia ecosistémica y los elementos conectores complementarios, de los cuales, hace parte el P.M.S.B., al integrar la red general de parques de
Bogotá, demostrando ser pieza esencial que contribuye, de manera significativa, en la regulación de condiciones ambientales y en la adaptación a la variabilidad climática.

Sistema antrópico: Está comprendido por el espacio público, lo observable en sus componentes y en los equipamientos construidos. El parque ha sido creado, con el fin de adecuarlo para satisfacer las necesidades de carácter recreativo, ambiental, educativo y paisajístico de la población.

Sistema funcional: Determinado por la conexión entre el entorno y los ciudadanos y podría hacer parte del sistema anterior y desempeñarse como sistema de menor jerarquía, pero al definir lo antrópico como "lo construido", resulta necesario diferenciar este sistema, como aquel en el que tiene injerencia el usuario. La clasificación de las funciones recae en percepciones que dependen, muchas veces, del modo de uso y el tipo de usuario. Para este caso, se desglosa en funciones más específicas: conservación natural, deportiva, cultura, recreación pasiva y entretenimiento.

Bases generadoras del modelo: Se detallaron procesos de integración, relaciones e interacciones externas e internas del sistema en cuestión, se moldeó la base para su manejo ambiental y se definieron, a pesar de su complejidad, de manera sencilla, las dinámicas que permiten el funcionamiento del P.M.S.B., como sistema:

Delimitación del sistema: Límites geográficos en el contexto urbano, como las vías de tránsito que dividen y emplazan al complejo P.M.S.B., así: Norte: calle 63 y carrera 68; Oriente: calle 64 y carrera 36A; Sur: calle 63, transversal 59A y calle 53; Occidente: Avenida 68, límite del costado suroccidental de la Unidad Deportiva el Salitre, límite del costado sur del Jardín Botánico junto a la Universidad Libre y Avenida Rojas.

Objetivo del sistema: La multifuncionalidad del P.M.S.B. hace que su propósito como sistema, se direccione a implicaciones naturales, sociales, culturales y económicas del ambiente urbano, por lo que el objetivo del sistema se establece así: "Optimizar de manera integral las funciones del parque, con énfasis en el manejo ambiental".

Elementos/características del sistema: Dado que ningún elemento se puede tomar de manera aislada, por lo que cada sistema interactúa constantemente con su entorno y demás sistemas, los atributos que permiten entender con mayor facilidad las interacciones existentes, entre el suprasistema y el sistema son:

Entradas: Son los insumos que recibe el complejo P.M.S.B. del ambiente urbano capitalino, para poder cumplir y mantener sus funciones; pueden ser de origen natural o artificial, como recursos naturales, humanos y financieros. 
Procesos: Son las transformaciones que sufren las entradas, con el fin de conservar componentes naturales y antrópicos del sistema.

Salidas: Son resultado de los procesos efectuados dentro del sistema y al igual que las entradas, hacen que este mantenga sus funciones evitando su deterioro.

Nivel jerárquico: Según la clasificación expuesta por Boulding (1956), el sistema del P.M.S.B. pertenece al octavo nivel, es decir, al sistema social o de organizaciones sociales, que destaca el rol social, interconectado por canales de comunicación, con personas capaces de compartir cultura, historia y otros factores.

\section{RESULTADOS Y DISCUSIÓN}

Interacciones entre elementos del sistema: Los componentes e interacciones son los escenarios que, al engranarse como subsistemas, mantienen la dinámica propia del sistema. Al mismo tiempo, la variación de rangos en los sistemas empieza a tomar forma y el sistema natural y antrópico de mayor jerarquía, a la hora de definir el macrosistema, pasa a hacer parte de los componentes esenciales del límite de interés, influyendo de manera directa en los subsistemas que conforman el P.M.S.B. Son partes que unidas hacen que funcione; actúan como un sistema de menor nivel jerárquico o subsistema, para este caso, corresponden a:

Jardín Botánico y Humedal El Salitre: Este subsistema tiene como objetivo la conservación natural, a partir de la promoción de la investigación, la educación y la recreación pasiva entre los usuarios. Su aporte al sistema radica en ser el escenario con mayor diversidad de especies de flora, además de aportar -como entidad-, que pueden aumentar o disminuir, de acuerdo a decisiones tomadas, el recurso humano, para el manejo del arbolado.

Centros deportivos (Unidad Deportiva El Salitre, Parque Recreo-Deportivo El Salitre, Complejo Acuático, Cancha pública Golf, Centro de alto rendimiento): Este componente cuenta con la mayor área de infraestructura para la práctica de deporte profesional y semiprofesional de diversas disciplinas. Su principal aporte está en los equipamientos deportivos.

Parque Central Simón Bolívar y Parque el Lago: Es el escenario más frecuentado para la recreación pasiva y la función paisajística; los espacios diversificados hacen que estos escenarios aporten a la integración de las dinámicas sociales.

Plaza de los Artesanos y Palacio de los Deportes: Escenarios especializados en albergar eventos culturales, empresariales y musicales, su principal aporte es la preponderancia social de los eventos, que atrae una buena cantidad de usuarios.

Salitre Mágico y Museo de los Niños: Son puntos de entretenimiento pago, contienen infraestructura mecánica para el disfrute de los usuarios. El aporte, se observa en la contribución a los recursos humanos y financieros internos.

Biblioteca Virgilio Barco: Se convierte en un centro cultural, brindando acceso al conocimiento; es una puerta pública a la información y los servicios, el aporte es la educación y los espacios.

Diseño del modelo: Se tuvieron en cuenta los esquemas de la modelación ODUM, que permitieron representar los elementos e interacciones integrantes del sistema. Se optó, en primera instancia, precisar en dos enfoques: uno, respecto al modelo de macrosistema ambiente urbano, que se observa en la figura 2 y otro, referido al modelo del sistema específico P.M.S.B., esquematizado en la figura 3. El primer diagrama muestra la relación entre el sistema natural y antrópico, elementos del macrosistema ambiente urbano, representados como depósitos o almacenes de biomasa (color azul), siendo simultáneamente productores de bienes y servicios (color verde), que son finalmente consumidos por los usuarios o habitantes de la ciudad (color naranja). El sistema funcional, se presenta como una interacción entre el entorno y los ciudadanos (color piel/carne) y tiene lugar cuando se requieren dos formas de energía, para simbolizar un componente diferente a los demás. Los flujos de energía inician con los recursos naturales y financieros (entradas, de color amarillo), ingresan a los depósitos y salen en forma de mejoras y energía degradada. El P.M.S.B. aparece como un productor en medio de estos dos depósitos, para destacar su emplazamiento dentro del macrosistema.

El segundo diagrama del sistema P.M.S.B. muestra los seis subsistemas que lo conforman, posicionados en forma cíclica, para ilustrar que se encuentran en el mismo nivel jerárquico; poseen el símbolo de productores y cada uno de ellos asienta depósitos de dinero, cuerpos de agua, espacios para erigir nuevas estructuras, entre otros, que pueden aumentar o disminuir, de acuerdo a las decisiones tomadas. Se representa, luego, otro tipo de productores de un nivel menor (verde oscuro), que simbolizan vegetación e infraestructura. Los usuarios, se ubican en el centro del modelo en constante relación y retroalimentación (flechas rojas) con estos componentes. Se representa también la relación creada entre los subsistemas y la avifauna como una interacción (Corredores de Avifauna, color dorado) y los flujos de dinero en líneas punteadas y ubicadas, además, en forma cíclica, al igual que el símbolo de interruptor (amarillo) que se genera, debido a la frecuencia de eventos y de conciertos que se realizan en el parque, los flujos de energía (entradas y salidas) son las mismas expuestas en el párrafo anterior. Finalmente, se dis- 


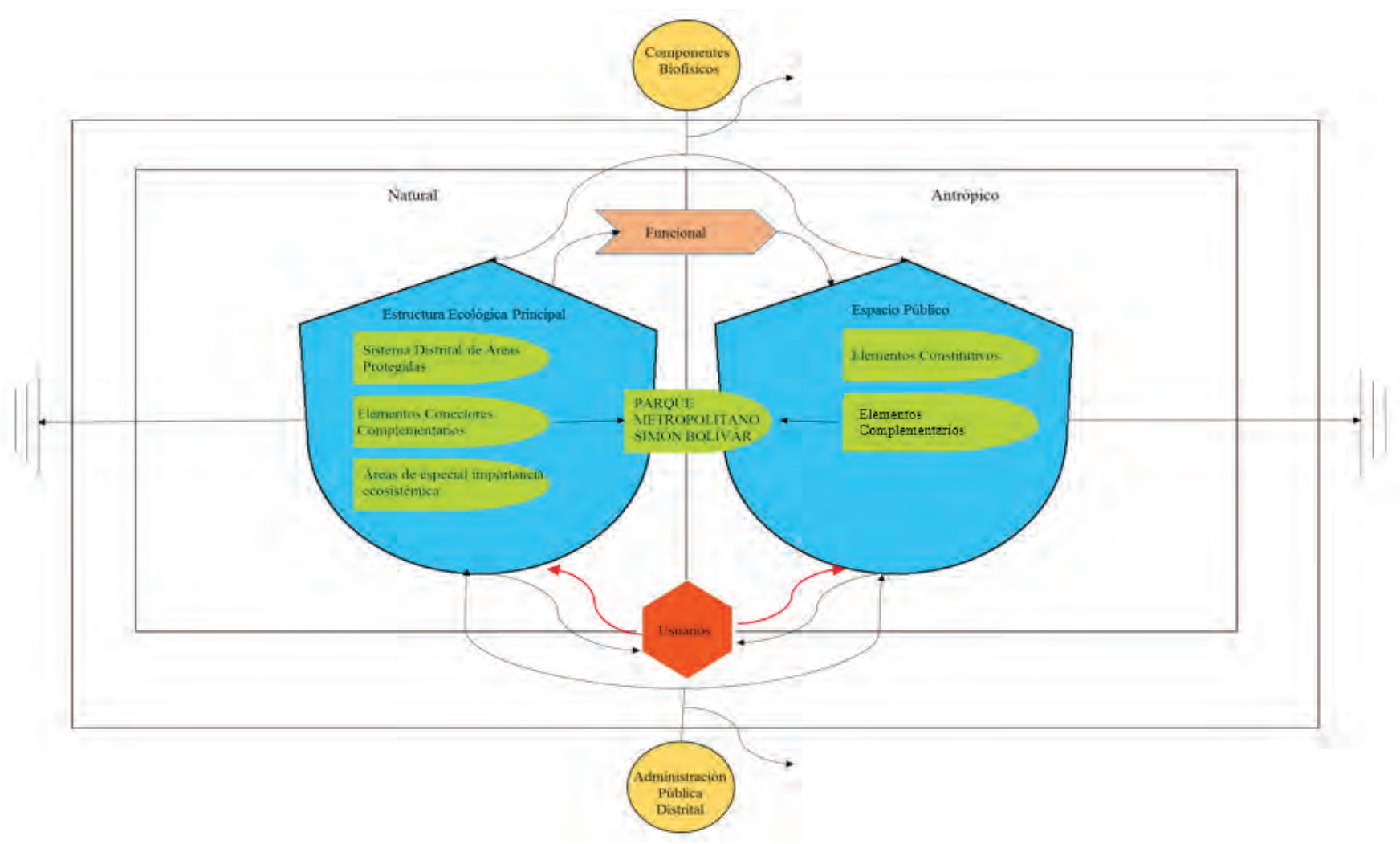

Figura 2. Modelo del macrosistema ambiente urbano. Elaborado por el segundo y el tercer autor del artículo.

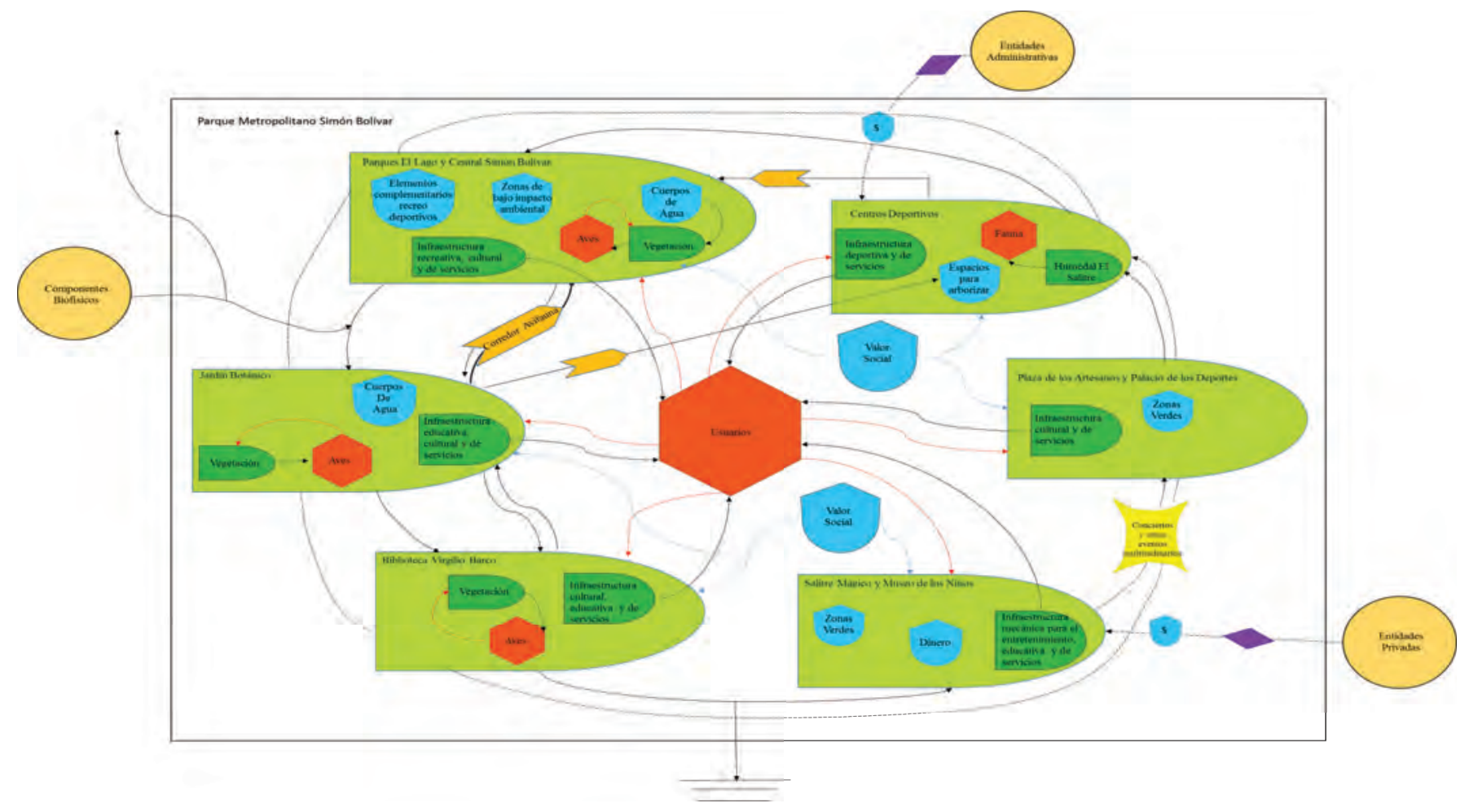

Figura 3. Modelo del sistema Parque Metropolitano Simón Bolívar. Elaborado por el segundo y el tercer autor del artículo. 
tingue el depósito "valor social", que abarca el sistema completo, como un resultado de los canales de comunicación concebidos en el modelo. También, se plasmaron las relaciones en un esquema real, expresado en la figura 4, teniendo en cuenta los siguientes lineamientos de importancia:

- El potencializar funciones, gira en torno al buen servicio para los usuarios, por ende, todos los esfuerzos van dirigidos a satisfacer sus necesidades, además la interacción es recíproca.

- La valoración social por parte del usuario hacia el parque es un aspecto prioritario para su conservación.

- La funcionalidad cultural es una oportunidad para difundir y promover el uso responsable de los escenarios.

- Los distintos escenarios pueden ser aptos para ofrecer una función diferente a la propia, dependiendo de la demanda de los usuarios.

- Los corredores ecológicos dependen no solo de interconectores naturales; los artificiales pueden contribuir de igual manera. Además, se pueden aprovechar espacios para aumentar la densidad arbórea.

- Siempre se requerirá de las entradas monetarias para mejorar las propiedades del parque; las entidades administrativas deben dotar con los medios necesarios para mantener en buen estado el parque.

Es así como el análisis sistémico puede apoyar la elaboración de unas acciones estratégicas, gracias a la posibilidad de observar al P.M.S.B., como una parte de un todo, en diferentes niveles espaciales $\mathrm{y}$, al mismo tiempo, como parte de un todo, que corresponde a distintos sistemas así:

- Sistema local social: la Localidad de Teusaquillo, en donde su mayor significancia está dada por corresponder a un área verde recreativa.

- Sistema urbano: la ciudad de Bogotá, en donde su mayor significancia está dada por ser el pulmón verde de la ciudad.

- Sistema municipal administrativo: el Distrito capital, en donde su mayor significancia está dada por ser parte importante del espacio público.

- Sistema regional ambiental: entre los cerros orientales y la cuenca alta del río Bogotá, en donde su mayor significancia está dada por ser parte de la subcuenca del Salitre, del entramado verde y de la red de humedales.

Entonces, en términos de escala espacial o de representatividad del área dentro de cada sistema, el P.M.S.B. tiene un mayor o menor porcentaje; por ejemplo, corresponde a un área grande dentro del sistema social-local, pero representa un área pequeña, dentro del sistema regional ambiental; sin embargo, su mayor importancia se resalta en términos de funcionalidad, ya que el P.M.S.B. es una parte bastante útil para todos los sistemas: el social, el urbano, el administrativo y el ambiental. Y aunque en ninguno de estos sistemas es el elemento núcleo, en su papel de elemento nodo, cumple una importante función para entretejer redes estructurantes, desde su potencial, como espacio público.

De lo anterior, se puede deducir, que las relaciones resultantes permiten entender la forma en que cada sistema constituye el elemento de un sistema más grande o jerárquicamente superior (Cadenas, 2005). Aquí, es donde se observa por qué esta teoría, aplicada al campo de la geografía, es denominada geografía sistémica, que con un enfoque integrador, ofrece la posibilidad de abordar el espacio geográfico, como un sistema con sus propiedades y características, fácil de esquematizar y de comprender (Popolizio, 1987), lo cual, se puede constatar con las interconexiones e integraciones de las funciones que presta cada escenario del sistema geográfico P.M.S.B. las que, a su vez, dependen de los aportes externos de las entidades distritales, de su entorno urbanizado, de la participación activa de los usuarios y de sus dinámicas recreativas, deportivas, culturales y de conservación natural. El macrosistema "Ambiente urbano de Bogotá", bien podría haber sido delimitado por el espacio público o la estructura ecológica principal; sin embargo, estos dos escenarios, se complementan uno a otro, por lo que resulta más práctico y se evita la exclusión de elementos influyentes para el parque, si se describen de manera conjunta.

Por otra parte, un modelo es una construcción de la realidad, que permite identificar relaciones complejas entre los componentes de un sistema (Arnold \& Osorio, 1998). El que se aplicó en este estudio, es el de diagramas de flujo, desarrollado por Odum (1988) y que concentra la importancia en los flujos de energía y retroalimentaciones (Mancera, 2003). El modelo es una representación esquemática de los elementos del sistema y las relaciones que existen entre ellos.

Desde la teoría, se evidencia que hay distintas concepciones del término manejo ambiental y en el contexto nacional, es usual que se defina como plan de manejo ambiental, entendido como las "medidas y actividades que, producto de la evaluación ambiental, están orientadas a prevenir, mitigar, corregir o compensar los impactos y efectos ambientales debidamente identificados, originados por el desarrollo de un proyecto, obra o actividad", pero teniendo en cuenta que este trabajo fue realizado en un parque urbano, que a diferencia de los parques nacionales o regionales de conservación natural en áreas con baja influencia urbana está rodeado de una matriz artificializada, fue necesario ajustar la definición y establecer que el manejo ambiental es "la aplicación de 


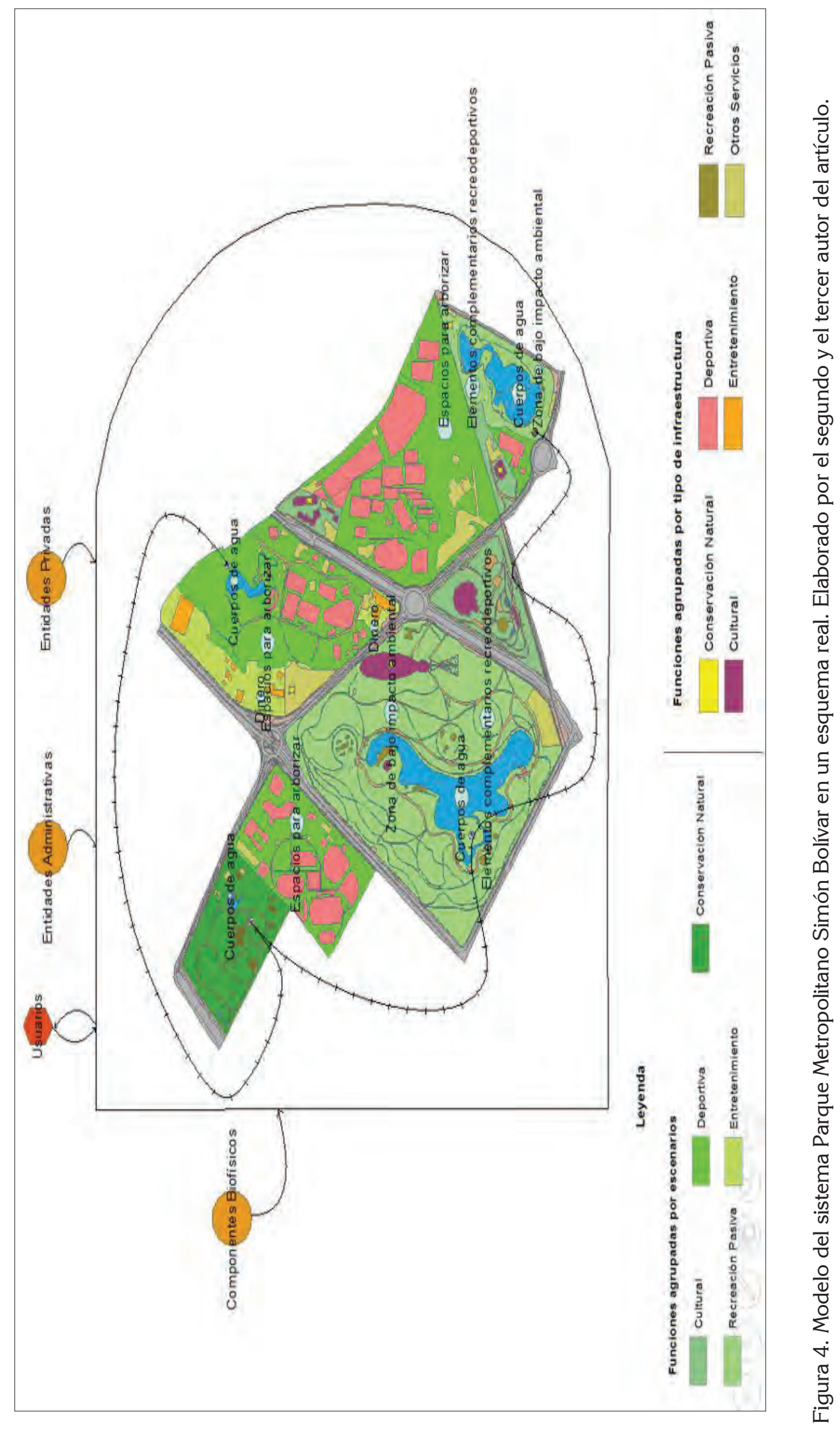


estrategias orientadas a la calidad de vida del ser humano, mientras se mejoran y se aprovechan espacios deteriorados, integrando factores ambientales, institucionales y sociales, en donde se cualifican las funciones de los parques, para generar zonificaciones que facilitan las acciones conducentes a su manejo integral".

En esa vía, Harnik (2010) hace un acercamiento a la planificación de sistemas verdes urbanos, combinando criterios económicos, sociales y ecológicos, para determinar su influencia, según los intereses públicos y privados de varias ciudades. Vélez (2007) considera que los parques urbanos deben ser espacios sustentables, con un mínimo impacto en los procesos de consumo energético, producción de desechos y artificialidad en su funcionamiento, con un manejo ambiental asociado a la reducción de pérdidas, cambio de hábitos de uso y consumo de recursos, optimización de procesos y reciclaje, entre otros; asimismo, estima la escala metropolitana como la más adecuada, para una óptima función de los parques.

Con la incorporación de estos conceptos y posiciones, se establece la importancia de aplicar modelos, que no subestimen las funciones de un parque metropolitano, sino por el contrario, ver este espacio natural, con ambientes artificiales, como una oportunidad para los ciudadanos, de comprender que hacen parte de un todo y si su uso no es el adecuado, se pierde la armonía del entorno, lo que, en consecuencia, redunda en el detrimento de las oportunidades, que puede ofrecer a la comunidad, para su calidad de vida. Para Rangel (s.f), las actitudes oficiales en ciudades latinoamericanas, para el tratamiento de un eje ambiental urbano integrado, generalmente, han sido contradictorias, por lo que cobra importancia el poder descubrir en la ciudad los elementos físicos y socioculturales de relevancia actual o potencial, para estructurar una red.

En Colombia, el MAVDT (2005) insta a impulsar mayor responsabilidad e interés social para que el espacio público sea revalorado como un lugar vital para el encuentro, la vida comunitaria y el mejoramiento de la calidad de vida. Específicamente en Bogotá D.C., a partir del Decreto 364, se clasificaron los parques urbanos, según su escala espacial, estando en primer lugar, los de la red general, conformados por los Parques Metropolitanos (área mayor a 10 hectáreas) y Zonales (área de 1 a 10 hectáreas); la estructura ecológica principal, se concibe como una red de espacios con alto valor ambiental, que proporcionan servicios ambientales y ecosistémicos, que buscan garantizar la sostenibilidad y la habitabilidad en la ciudad (AMB, 2013).
Según Posada (2014), la urgencia de hacer de Bogotá un territorio más armónico, se destaca no solamente por ser la capital colombiana, sino por contar con ventajas comparativas en su ubicación, en la conformación de su estructura natural, en la responsabilidad de y para más de siete millones de personas y en la administración de recursos financieros, que se deben invertir en el mejoramiento de las condiciones actuales. En particular, en la evaluación del cumplimiento de las funciones ambientales de los parques metropolitanos de Bogotá D.C, a través de indicadores definidos ecológica y culturalmente, Franco (2013) concluyó que están cerca de cumplir sus funciones, aunque el componente ecológico es el que presenta menor grado de cumplimiento, pero la integración de los usuarios al diseño ambiental específico de los proyectos urbanos, es una estrategia escasamente aplicada, que ofrece un amplio potencial de aplicación en la mejora del entorno colectivo (Puyuelo \& Gual, 2009).

Para lograr la gestión ambiental urbana, Zúñiga \& Pérez (2013) destacan tres aproximaciones: la conservación integrada o ecosistémica, el enfoque estratégico y un enfoque participativo. Las tres perspectivas deben ser parte de un proceso de mejora continua, que desarrolle prioridades e intereses consensuados en una visión prospectiva del desarrollo local, lo que es corroborado por Sanabria (2012), quien enfatiza en la importancia de tener en cuenta las condiciones locales, para definir las alternativas de diseño del espacio público en la ciudad y, por otra parte, corroborando lo que afirman Pinzón \& Echeverri (2010), el compromiso ciudadano permite tejer relaciones de armonía y de cooperación y al recuperar elementos de gran valor y potencial ambiental, cultural y recreativo, existentes en la ciudad, como lo son los parques de gran tamaño.

Con el ejercicio anterior, se concluye sobre la necesidad de integrar un manejo ambiental en los parques urbanos y, específicamente, bajo un enfoque sistémico, que permita recomponer, de manera gradual, el entramado socioambiental. Entonces, para no limitar las acciones al mantenimiento del P.M.S.B. y poder administrarlo con énfasis en la coordinación integral de funciones, se sugiere considerar las siguientes acciones estratégicas, a desarrollar de manera gradual en el corto, mediano y largo plazo (Tabla 1).

Conflicto de intereses: El manuscrito fue preparado y revisado con la participación de todos los autores, quienes declaramos que no existe conflicto de intereses que ponga en riesgo la validez de los resultados presentados y agradecen a la U.D.C.A, la financiación de la investigación "Convergencias de la Gestión Ambiental Territorial en Bogotá". 
Tabla 1. Propuesta de acciones estratégicas graduales para el manejo ambiental del Parque Metropolitano Simón Bolívar, 2016.

\begin{tabular}{|c|c|c|c|}
\hline $\begin{array}{l}\text { SISTEMAS EXTERNOS DEL } \\
\text { PMSB /TEMPORALIDAD DE LAS } \\
\text { ACCIONES ESTRATÉGICAS }\end{array}$ & CORTO PLAZO & MEDIANO PLAZO & LARGO PLAZO \\
\hline LOCALIDAD URBANA & $\begin{array}{l}\text { Propiciar la generación de } \\
\text { información sobre el PMSB } \\
\text { y comunicarla. Activar la } \\
\text { participación ciudadana en el } \\
\text { plan de manejo ambiental. }\end{array}$ & $\begin{array}{l}\text { Mejorar el conocimiento de } \\
\text { las formas de integración } \\
\text { urbana. } \\
\text { Introducir, en conjunto con } \\
\text { la comunidad, medidas de } \\
\text { aumento de la biodiversi- } \\
\text { dad. }\end{array}$ & $\begin{array}{l}\text { Transformar y mejorar los } \\
\text { elementos del sistema, } \\
\text { con grupos voluntarios y } \\
\text { en coordinación interin- } \\
\text { stitucional. }\end{array}$ \\
\hline CIUDAD /MALLA URBANA & $\begin{array}{l}\text { Integrar al PMSB a la pla- } \\
\text { neación y ordenación ur- } \\
\text { bana, con miras a conformar } \\
\text { una "ciudad verde". }\end{array}$ & $\begin{array}{l}\text { Involucrar a agentes } \\
\text { económicos y univer- } \\
\text { sidades para aplicar } \\
\text { soluciones y tecnologías } \\
\text { verdes, en los anillos que } \\
\text { rodean al PMSB. }\end{array}$ & $\begin{array}{l}\text { Introducir sistemas de } \\
\text { mejora de la cogestión } \\
\text { socioambiental, con in- } \\
\text { versión privada. }\end{array}$ \\
\hline $\begin{array}{l}\text { PERIURBANO y } \\
\text { DISTRITO CAPITAL }\end{array}$ & $\begin{array}{l}\text { Promocionar las ventajas de } \\
\text { preservar el tejido natural del } \\
\text { centro a la periferia y vice- } \\
\text { versa, para motivar la agri- } \\
\text { cultura urbana y la relación } \\
\text { con la agricultura rural del } \\
\text { Distrito. }\end{array}$ & $\begin{array}{l}\text { Integrar el PMSB con énfa- } \\
\text { sis en el plan de manejo de } \\
\text { la microcuenca El Salitre. }\end{array}$ & $\begin{array}{l}\text { Consolidar las redes } \\
\text { ecológicas funcionales in- } \\
\text { tegradas por los espacios } \\
\text { protegidos, los humedales } \\
\text { y las zonas de importan- } \\
\text { cia ecológica de Bogotá. }\end{array}$ \\
\hline $\begin{array}{l}\text { ESTRUCTURA ECOLÓGICA } \\
\text { PRINCIPAL REGIONAL }\end{array}$ & $\begin{array}{l}\text { Generar, de manera interin- } \\
\text { stitucional, políticas ecour- } \\
\text { banísticas y ecoturísticas de } \\
\text { complementariedad donde } \\
\text { PMSB, juegue un papel im- } \\
\text { portante. }\end{array}$ & $\begin{array}{l}\text { Identificar y valorar los } \\
\text { bienes y servicios ecosis- } \\
\text { témicos, en los cuales, el } \\
\text { PMSB, aporta su parte. }\end{array}$ & $\begin{array}{l}\text { Desarrollar un programa } \\
\text { de seguimiento, veeduría } \\
\text { y control ciudadano, para } \\
\text { que la conectividad eco- } \\
\text { sistémica se mantenga y } \\
\text { mejore. }\end{array}$ \\
\hline
\end{tabular}

\section{BIBLIOGRAFÍA}

1. ALCALDÍA MAYOR DE BOGOTÁ D.C. -AMB-. 1988. Decreto 1504 de Nivel Nacional. Disponible desde internet en: http://www.alcaldiabogota.gov.co/ sisjur/normas/Norma1.jsp?i=1259. (con acceso 25/09/2015).

2. ALCALDÍA MAYOR DE BOGOTÁ D.C. -AMB-. 2010. Decreto 360. Disponible desde internet en: http:// www.alcaldiabogota.gov.co/sisjur/normas/Norma1. jsp?i=1259. (con acceso 25/09/2015).

3. ALCALDÍA MAYOR DE BOGOTÁ D.C. -AMB-. 2013. Decreto 364. Disponible desde internet en: http:// www.sdp.gov.co/portal/page/portal/PortalSDP/actualidad-SDP-home/Decreto-364-baja.pdf (con acceso 28/01/2016).
4. ÁNGEL M., A.; VELÁSQUEZ B., L.S. s.f. El medio ambiente urbano acercamientos conceptuales. Disponible desde internet en: http://www.biociudades.org/files/AUGUSTO-LUZ-8-.pdf (con acceso 28/01/2016).

5. ARNOLD, M.; OSORIO, F. 1998. Introducción a los conceptos básicos de la Teoría General de sistemas. Cinta moebio 3:40-49.

6. BOULDING, K.E. 1956. La teoría general de sistemas: La estructura interna de la ciencia. N.W. Botero D. (traductor). 2007. Politécnica, 4:103-115.

7. CADENAS M., A. 2005. La economía ecológica como ciencia del Desarrollo Sostenible. Encuentros Multidisciplinares. 7(20):32-39.

8. CARDONA A., O. 1996. Manejo ambiental y prevención de desastres: dos temas asociados. Ciudades en 
riesgo degradación ambiental, riesgos urbanos y desastres. Ed. USAID. Ecuador. p 59-76.

9. CENTRO DE ESTUDIOS AMBIENTALES -CEA-. 2014. La infraestructura verde urbana de Vitoria-Gasteiz. País Vasco. 94 p. Disponible desde internet en: http://www.vitoria-gasteiz.org/wb021/http/contenidosEstaticos/adjuntos/eu/32/95/53295.pdf (con acceso $26 / 02 / 2016$ ).

10. FERNÁNDEZ, R. 2002. La ciudad verde: teoría de la gestión ambiental urbana. Espacio Editorial. Argentina. 517p.

11. FRANCO C., J. 2013. Evaluación del cumplimiento de las funciones ambientales de los parques urbanos de Bogotá D.C. Pontificia Universidad Javeriana, Bogotá D.C. Disponible desde internet en: http://repository.javeriana.edu.co/handle/10554/12384 (con acceso 25/07/2015).

12. GARCÍA L., M. 1989. El parque urbano como espacio multifuncional. Paralelo 37. Estudios Geográficos. España. 13:105-111.

13. GARCÍA H., S.; GUERRERO, M. 2006. Indicadores de sustentabilidad ambiental en la gestión de espacios verdes. Parque urbano Monte Calvario, Tandil, Argentina. Rev. Geografía Norte Grande. 35:45-57.

14. GÓMEZ L., F. 2005. Las zonas verdes como factor de calidad de vida en las ciudades. Ciudad y Territorio Estudios Territoriales. España. 144:417-436.

15. HARNIK, P. 2010. Planificación de parques urbanos: la experiencia estadounidense. Economía, Sociedad y Territorio. México. 11(37):795-801.

16. INFRAESTRUCTURA DE DATOS ESPACIALES PARA EL DISTRITO CAPITAL -IDECA-. 2015. Infraestructura de datos espaciales para el distrito capital. Disponible desde internet en: http://mapas.bogota.gov.co/ portalmapas/ (con acceso 25/06/2015).

17. MALDONADO, A. 1999. La construcción de indicadores Bio-Ecológicos para medir la calidad del ambiente natural urbano. Documento de investigación del Grupo de Calidad Ambiental Urbana de la Facultad de Arquitectura y Arte de la Universidad de Los Andes. Venezuela. Disponible desde internet en: http:// www.perfilciutat.net/fitxers/IVSL_A2.pdf (con acceso 25/11/2015).
18. MANCERA P., J. 2003. Introducción a la modelación ecológica. Principios y aplicaciones. Colombia._Disponible desde internet en: https://books.google. com.co/books?id =-AfcTs-AtvUC\&printsec $=$ frontc over\&hl $=\mathrm{es} \# \mathrm{v}=$ onepage $\& \mathrm{q} \& \mathrm{f}=$ false. $\quad$ (con acceso 25/07/2015).

19. MINISTERIO DE AMBIENTE, VIVIENDA Y DESARROLLO TERRITORIAL -MAVDT-. 2008. Política de Gestión Ambiental Urbana. Colombia._Disponible desde internet en: https://www.minambiente.gov.co/index. php/component/content/article?id=2050:plantillaasuntos-ambientales-y-sectorial-y-urbana-sin-galeria-84\#documentos-relacionados (con acceso 25/09/2015).

20. MINISTERIO DE AMBIENTE, VIVIENDA Y DESARROLLO TERRITORIAL -MAVDT-. 2005. Guía de Mecanismos de Recuperación del Espacio Público. Serie Espacio Público \#5. Colombia. 64p.

21. ODUM P., H. 1988. Energy, environment and public policy. A guide to the analysis of systems. Disponible desde internet en: http://www.unep.org/regionalseas/ publications/reports/RSRS/pdfs/rsrs095.pdf (con acceso 25/07/2015).

22. ORGANIZACIÓN MUNDIAL DE LA SALUD -OMS-. 1965. Cuestiones de higiene los medios relacionados con la ordenación urbana y la urbanización. Informe técnico \#297. Ginebra. 74 p. Disponible desde internet en: http://apps.who.int/iris/bitstream/10665/37718/1/ WHO_TRS_297_spa.pdf (con acceso 28/01/2016).

23. PINZÓN B., M.V.; ECHEVERRI A., I.C. 2010. Espacio Público, cultura y calidad ambiental urbana, una propuesta metodológica para su intervención. Invest. Desarrollo. Colombia. 18(1):92-113.

24. POPOLIZIO, E. 1987. El enfoque sistémico en la enseñanza de la Geografía. Boletín de la Sociedad Argentina de Estudios Geográficos G/EA N¹06 Buenos Aires.

25. POSADA A., A. 2014. De la planeación a la gestión ambiental colectiva: posibilidades en Bogotá-Colombia. Rev. U.D.C.A Act \& Div. Cient. 17(1):257-264.

26. PUYUIELO, M.; GUAL, J. 2009. Diseño prospectivo y elementos de uso en parques urbanos a partir de la experiencia de las personas mayores. Medio Ambiente y Comportamiento Humano. 10(1y2):137-160. 
27. RANGEL M., M. (s.f.). Redes de espacios públicos y ejes ambientales estructurantes. Disponible desde internet en: http://www.saber.ula.ve/bitstream/123456789/17245/1/redes_spacio.pdf (con acceso 29/01/2016).

28. SANABRIA A., T. 2012. La aplicación de la ecología del paisaje al espacio público de las ciudades Colombianas. Revista electrónica p.120-149. http://www. uniboyaca.edu.co/facultades/FABA/designia (con acceso 29/01/2016).
29. VÉLEZ R., A. 2007. La conservación de la naturaleza urbana un nuevo reto en la gestión ambiental de las ciudades, para el siglo XXI. Bitácora Urbano Territorial. Colombia. 11(1):20-27.

30. ZÚINIIGA, L.; PEREZ, R. 2013. Los recursos construidos de valor patrimonial en un modelo de gestión ambiental urbana. EURE 39(117):69-90

Recibido: Enero 27 de 2016

Aceptado: Marzo 28 de 2016

Cómo citar:

Posada Arrubla, A.; Paredes Buitrago, A.D.; Ortiz Romero, G.E. 2016. Enfoque sistémico aplicado al manejo de parques metropolitanos, una posición desde Bogotá D.C. - Colombia. Rev. U.D.C.A Act. \& Div. Cient. 19(1): 207-217. 